\title{
Hemorrhage into the gallbladder caused by pseudoaneurysm of the cystic artery
}

A 77-year-old woman was admitted to hospital with cholecystitis. After 8 days of antibiotic treatment she developed hypotension, weakness, and anemia. Abdominal computed tomography showed a tense gallbladder containing blood and a round nodule which enhanced after administration of contrast material (Figure 1). Doppler ultrasonography showed a nodule with arterial flow and a "ying yang" pattern (Figure 2 ), and selective angiography revealed an cystic artery pseudoaneurysm (Figure 3). Metallic coils were used as embolization material and full occlusion of the aneurysmal sac was achieved.

Hemorrhage into the gallbladder occurs rarely and can result from gangrenous cholecystitis, cystic artery pseudoaneurysm, varicose veins in the gallbladder wall, or tumors [1]. In this case hemorrhage was caused by a pseudoaneurysm. Cystic artery pseudoaneurysms can form secondary to atherosclerosis, or can follow trauma; they can also arise as a result of arterial wall erosion by local inflammatory processes such as cholecystitis $[2,3]$. The diagnosis is difficult but it may be suggested by abdominal computed tomography and Doppler ultrasonography. Angiography allows for both a definitive diagnosis and simultaneous therapeutic intervention [4].

Endoscopy_UCTN_Code_CCL_1AZ_2AN

\section{J. L. Pérez-Castrillón', M. Mendo², H. Calero ${ }^{2}$}

${ }^{1}$ Department of Medicine, University Hospital Río Hortega, Valladolid, Spain

2 Department of Radiology, University Hospital Río Hortega, Valladolid, Spain.

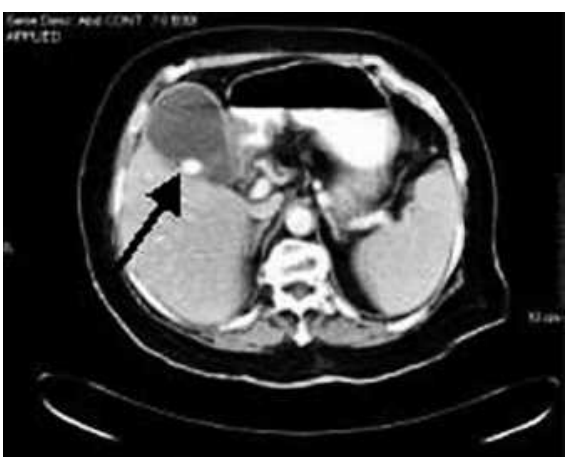

Figure 1 Abdominal computed tomography showed blood and a nodule in the gallbladder.

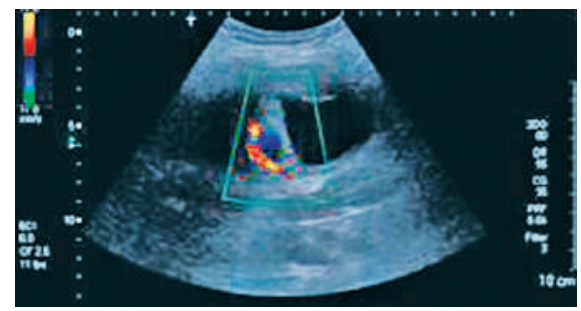

Figure 2 Doppler ultrasonography showed a nodule with a "ying yang" pattern.

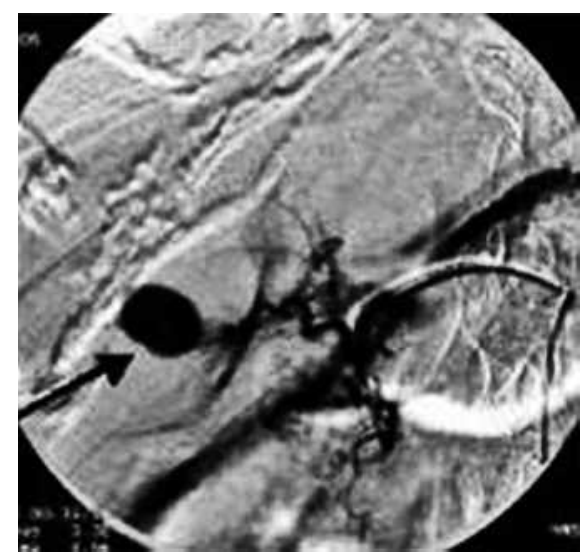

Figure 3 Selective angiography revealed a cystic artery pseudoaneurysm (arrow).

\section{References}

${ }^{1}$ Berland LL, Doust BD, Foley WD. Acute hemorrhage into the gallbladder diagnosed by computed tomography and ultrasonography. J Comput Assist Tomogr 1980; 4: 260-262

${ }^{2}$ Gutierrez C, Ramia JM, Villar J et al. Cystic artery pseudoaneurism from an evolved acute calculous cholecystitis. Am J Surg 2004; 187: 519-520

${ }^{3}$ Siablis D, Papathanassiou ZG, Karnabatidis D et al. Hemobilia secondary to hepatic artery pseudoaneurysm: an unusual complication of bile leakage in a patient with a history of a resected IIIb Klatskin tumor. World J Gastroenterol 2005; 11: 5229-5231

${ }^{4}$ Moodley J, Singh B, Lalloo S et al. Non-operative management of haemobilia. $\mathrm{Br} \mathrm{J}$ Surg 2001; 88: $1073-1076$

Corresponding author

\section{J. L. Pérez-Castrillón}

University Hospital Río Hortega

Cardenal Torquemada s/n

47010 Valladolid

Spain

Fax: $\quad$ +34-983-331566

E-mail: castrv@terra.es 